\title{
EVALUATING THE SOCIAL AND CULTURAL IMPLICATIONS OF THE INTERNET
}

\author{
Philip Brey \\ Department of Philosophy \\ University of Twente
}

The Netherlands

\section{$1 \quad$ INTRODUCTION}

Since the Internet's breakthrough as a mass medium, it has become a topic of discussion because of its implications for society. At one extreme, one finds those who only see great benefits and consider the Internet a tool for freedom, commerce, connectivity, and other societal benefits. At the other extreme, one finds those who lament the harms and disadvantages of the Internet, and who consider it a grave danger to existing social structures and institutions, to culture, morality and human relations. In between one finds the majority, those who recognize both benefits and harms in the Internet as it currently exists and who recognize its usefulness while worrying about some of its negative impacts.

As an example of a positive appraisal of the Internet, consider what Esther Dyson, one of the early enthusiasts for the Internet, states in her book Release 2.0. There, she claims: "The Net offers us a chance to take charge of our own lives and to redefine our role as citizens of local communities and of a global society. It also hands us the responsibility to govern ourselves, to think for ourselves, to educate our children, to do business honestly, and to work with fellow citizens to design rules we want to live by." (Dyson, 1997). Dyson argues that the Internet offers us the chance to build exciting communities of likeminded individuals, enables people to redefine their work as they see fit, fosters truth-telling and information disclosure, helps build trust between people, and can function for people as a second home.

For a negative appraisal, consider the opinion of the Council of Torah Sages, a group of leading orthodox rabbis in Israel who in 2000 issued a ruling banning the Internet from Jewish homes. The Council claimed that the Internet is "1,000 times more dangerous than television" (which they banned thirty years earlier). The Council described the Internet as "the world's leading cause of temptation" and "a deadly poison which burns souls" that "incites and encourages sin and abomination of the worst kind." The Council explained that it recognized benefits in the Internet, but saw no way of balancing these with the potential cost, which they defined as exposure to "moral pollution" and possible addiction to Internet use that could quash the motivation to learn Torah, especially among children. ( See Ha'aretz, January 7, 2000.)

Even the greatest critics of the Internet, like the Council of Torah Sages, see benefits in the technology, and even the greatest advocates recognize that there are drawbacks to the medium. People have different opinions on what the benefits and disadvantages are and also differ in the way in which they balance them against each other. Underlying these different assessments of the Internet are different value systems. Esther Dyson holds a libertarian value system in which the maximization of individual freedom, property rights and free market capitalism are central values. Her positive assessment of the Internet is based on the potential she sees in this technology to promote these values. In contrast, the values Council of Torah Sages are values of Hareidi, a variety of orthodox Judaism, according to which the highest good is obedience to God's law as laid out in the Torah, and they concluded, based on these values, that the Internet is harmful.

Yet, it is not just differences in value systems that determine one's appraisal of a technology like the Internet. Such an appraisal is also determined by one's empirical understanding of how the technology works and what its consequences

SIGCAS Computers and Society

Volume 36, No. 3, September 2006 
or implications are. People often come to unduly positive or negative appraisals of technology because they assess its consequences wrongly. For instance, some people believe that Internet use increases the likelihood of social isolation, but empirical research could conceivably show that in fact the opposite is the case. Disagreements about the positive and negative aspects of the internet may therefore be either normative disagreements (disagreements about values) or empirical disagreements (disagreements about facts). Of course, it is not always easy to disentangle values and empirical facts, as these are often strongly interwoven.

N ext to contested benefits and harms of the Internet, there are also perceived harms and benefits that are fairly broadly acknowledged. For instance, nearly everyone agrees that the Internet has the benefit of making a large amount of useful information easily available, and nearly everyone agrees that the Internet can also be harmful by making dangerous, libelous and hateful information available. People have shared values and shared empirical beliefs by which they can come to such collective assessments.

M y purpose in this essay is to contribute to a better understanding of existing positive and negative appraisals of the Internet, as a first step towards a more methodical assessment of Internet technology. My focus will be on the appraisal of social and cultural implications of the Internet. Whether we like it or not, policy towards the Internet is guided by beliefs about its social and cultural benefits and harms. It is desirable, therefore, to have methods for making such beliefs explicit in order to analyze the values and empirical claims that are presupposed in them.

In the next two sections (2 and 3), I will catalogue major perceived social and cultural benefits and harms of the Internet, that have been mentioned frequently in public discussions and academic studies. I will focus on perceived benefits and harms that do not seem to rest on idiosyncratic values, meaning that they seem to rest on values that are shared by most people. For instance, most people believe that individual autonomy is good, so if it can be shown that a technology enhances individual autonomy, most people would agree that this technology has this benefit. Notice, however, that even when they share this value, people may disagree on the benefits of the technology in question, because they may have different empirical beliefs on whether the technology actually enhanced individual autonomy.

Cataloguing such perceived cultural benefits and harms is, I believe, an important first step towards a social and cultural technology assessment of the Internet and its various uses. A n overview of perceived benefits and harms may provide a broader perspective on the Internet that could be to the benefit of both friends and foes, and can contribute to a better mutual understanding between them. M ore importantly, it provides a potential starting point for a reasoned and methodical analysis of benefits and harms. Ideas on how such an analysis may be possible, in light of the al ready mentioned facts that assessments are based on different value systems, will be developed in section 4 . In a concluding section, I sketch the prospects for a future social and cultural technology assessment of the Internet.

\section{PERCEIVED BENEFITS OF THE INTERNET TO CULTURE AND SOCIETY}

The benefits of the Internet that are mentioned most frequently are its perceived benefits as a means to information, communication, commerce, entertainment, and social interaction. These are all functional aspects of the internet: most of its applications and services have been designed explicitly to serve such functions, and many people that the Internet successfully performs these functions. In addition, the Internet has been claimed to have benefits that are less intentional: benefits to individual development and cultural understanding, particularly. The following list of major perceived benefits is suggestive but not exhaustive:

1. Access to information. The Internet makes a vast amount of information available, from a plurality of information sources, and makes it continuously available, more or less independent of time and place. A dequate information is of major importance to the successful functioning of (modern) individuals, and therefore any enhancement of the ability to acquire or access information can be seen as a great benefit to society.

2. Information dissemination. The Internet makes it possible for anyone to quickly, easily and inexpensively post and disseminate information and make this information available to a large audience. In this way, the Internet promotes freedom of speech by enhancing the ability of individuals to voice opinions and inform and influence others, which can be considered a great benefit.

3. Communication. The Internet facilitates one-to-one, one-to-many and many-to-many communication and enables users to communicate easily and inexpensively with a wide variety of individuals across the globe. 
Communication goes beyond the dissemination of information: it is a two-way process that allows for the expression of viewpoints, the creation of intimacy, and the coordination of actions. Because communication is so important to individuals, the Internet's enhancement of the power to communicate can be considered a great benefit.

4. Developing and maintaining social relations. The internet facilitates the development and maintenance of social relations with people outside one's immediate vicinity, and provides added means to maintain relations with people in one's vicinity. Social relations are very important to the functioning of individuals and of society as a whole, and the Internet provides powerful means for developing and maintaining such relations, which is a great benefit.

5. Community formation and social organization. The internet facilitates the development and maintenance of communities of individuals with shared interests and concerns and the formation and maintenance of structured organizations with specific agendas. The Internet also provides new ways for individuals to engage in collective behavior and form social movements. Community formation and social organization are important in any society, and any technology that provides new means to support these processes can be seen to provide important benefits.

6. Production and Commerce. The Internet enables new models for production by enabling coordination and partial automation of productive processes that span time and space. The Internet also enables new models for commerce, trade and business. These economic benefits translate into social and cultural benefits because they provide people with new products and services and faster and easier delivery of existing products and services at less cost.

7. Leisure and entertainment. The Internet enables new forms of leisure and entertainment, both for individual use and in interaction with others, such as playing games and providing forums for collectively practicing hobbies and for sharing and trading cultural objects like pictures, stories, drawings, software, music, and video.

8. Identity formation and psychological development. The Internet has been claimed to have positive effects on identity formation and psychological development by allowing people to experiment with alternative identities, to hide aspects of their identity that could meet with disapproval or stereotyping in face-to-face situations, to reveal aspects of themselves in relative anonymity, that they would not reveal in real life, and to expose themselves to a very broad variety of views and opinions (Turkle, 1995; Rheingold, 2000).

9. Learning and cognitive development. The Internet has been claimed to have beneficial effects on learning and cognitive development. It has been claimed that the Internet, and computers more generally, support interactive learning styles, enhances learning by supporting new multimedial ways of presenting information, and the development of good sensorimotor abilities (V an Dijk, 1999, p. 204-6).

10. Cultural understanding. It has been claimed that the Internet can promote a better understanding between cultures and cultural identities by enabling people from different cultural backgrounds and with different social and cultural identities to come together and communicate with each other under conditions that are conducive to cultural exchange (Ess and Sudweeks, 2001).

\section{PERCEIVED HARMS OF THE INTERNET TO CULTURE AND SOCIETY}

Some of the harms of the Internet that are mentioned frequently run directly contrary to their perceived benefits. Without denying that the internet provides new possibilities for information, communication, commerce, entertainment, and social interaction, critics tend to argue that the quality of these activities is harmed when they are performed over the Internet. So there is a comparative harm: compared to traditional ways of performing these activities, the Internet offers us an inferior or less beneficial way of performing them. In addition, critics perceive a number of other negative consequences of Internet use, that concern human development, human experience and social organization.

1. Information overload. It has been claimed that the Internet contributes to information overload, by stimulating the consumption of information and exposing users to an abundance of often irrelevant, unclear and inaccurate messages and data fragments. This has been claimed, for individuals, to cause anxiety, poor decision-making, difficulties in memorizing and remembering, and reduced attention span, and, for organizations, to lead less efficiency and poorer decision-making ( Edmunds and M orris, 2000). 
2. False information. It has been claimed that, because of the absence of good selection mechanisms, the Internet contains much false and unreliable information. Moreover, it is often impossible to evaluate the correctness of information found on the Internet, as it is often difficult to credit or evaluate sources. Therefore, the Internet is thought to represent a step backwards compared to more traditional information media.

3. Harmful information. It has been claimed that, because of the absence of selection mechanisms and effective regulation, the Internet contains a lot of harmful information: extremist ideology, recipes for making bombs, extreme forms of pornography, libelous information, and so forth. Compared to other, more controlled information media, the Internet can therefore be thought to do more harm.

4. Harmful communication. It has been claimed that conversations and dialogues on the internet often contain disrespectful, hurtful and inflammatory speech, and that conversations are often trivial or vulgar. The Internet has also been claimed to facilitate unwanted communication and harassment. Compared to other forms of communication, the Internet can therefore be claimed to represent a step backwards.

5. Harmful effects on social relations. It has been claimed that online social relationships may indirectly hurt offline (face-to-face) social relationships, because people will take less time and make less effort to engage in face-to-face interaction. It has also been claimed that online social relationships tend to be less profound, valuable and durable than offline relationships, and that computer-mediated communication less expressive and authentic than face-to-face communication (D reyfus, 2001)

6. Harmful effects on community and social organization. It has been claimed that the emergence of virtual communities and organizations may hurt existing "physical" communities and organizations because people will devote less time and energy to them, and that virtual communities and organizations are less profound, valuable and durable than "physical" ones (W inner, 1997; B orgmann, 2004).

7. Harmful effects on production and commerce. It has been argued that the easy distribution of digital information over the Internet will hurt commerce and the so-called culture industry (musicians, film makers, artists, etc.). It has also been claimed that the Internet, and digital media generally, negatively affect artistic production by rationalizing art and art production techniques. A nother claim is that Internet commerce is vulnerable to theft, fraud and hacking.

8. Harmful effects on identity formation and psychological development. It has been claimed that the relative anonymity (or pseudonymity) by which actions can be performed in cyberspace can lead to antisocial behavior that is performed without retribution. Similarly, computer games enable violent and disrespectful behavior that is often left unpunished, and may even rewarded. The possibility of such consequenceless antisocial behaviors is held to harm psychological development (Dreyfus, 2001; M cCormick, 2001). Also, the ready and availability of Internet pornography, that can be consumed relatively anonymously, has been claimed to promote a harmful form of disinhibition that harms personalities. M ore generally, Internet can lead to addiction (Y oung, 2004).

9. Harmful effects on learning and cognitive development. The Internet has been claimed to harm learning and cognitive development by offering an associationist rather than a discursive model of knowledge (i.e. hypertext and hypermedia rather than traditional types of texts), by disinhibiting autonomous knowledge production ("copy-paste mentality"), by limiting the attention span of cognitive subjects ("zap culture,") and by disinhibiting the critical appraisal of information (the absence of accountability for online information) (Dreyfus, 1999).

10. Cultural fragmentation. It has been claimed that the Internet promotes cultural fragmentation by stimulating the formation of virtual communities and groups organized around specialized interests, themes or cultural identities (W inner, 1997).

11. Loss of the sense of reality. It has been claimed that the Internet helps eradicate the distinction between reality and representation, and creates a constant confusion about this distinction, leading to insecurities, disagreements, and a loss of meaning (Baudrillard, 1995; B orgmann, 1999).

12. Loss of privacy and private-public boundaries. It has been argued that there is little privacy on the Internet, and that it is difficult to maintain clear boundaries between public and private spaces on the Internet, with resulting insecurities about the privacy conditions under which users operate. 


\section{ANALYZING AND EVALUATING BELIEFSABOUT THE INTERNET'S BENEFITSAND HARMS}

How could statements about harms or benefits of the Internet be assessed for their validity? I will argue that such assessments are only possible to an extent. It is possible to analyze statements and critique them, but it is not possible to validate or refute them conclusively, because they rest in part on (subjective) values. A distinction can be made between descriptive analysis and critical analysis. Descriptive analysis merely tries to understand statements by analyzing their meaning and presuppositions. Such an analysis would consider the context in which the statement is made and then analyze the values and empirical claims presupposed in the statement. In addition, it would analyze the meaning of the concepts used in the statement, since concepts may be used with different meanings. Descriptive analysis would thus involve four steps: (1) clarification of the meanings of concepts in the statement; (2) identification of presupposed values; (3) identification of implied empirical claims; (4) examination of the evidence for implied empirical claims.

A critical analysis goes beyond a descriptive analysis in that it would also proceed to examine the empirical evidence for these empirical claims and, optionally, would critique the values on which the statement rests and the soundness and clarity of the concepts used in the statement. Critical analysis would thus involve an additional fifth step: (5) critical assessment of values and concepts.

To see how a critical analysis of statements about the Internet could proceed, let us consider A lbert Borgmann's belief that cyberspace presents an escape into an alternative reality that is illusory because it presents us with trivialized and glamorized substitute realities that lack context (Borgmann, 1999, p. 191-2 -not literally Borgmann's statement but summarizes his position). Taking this statement's context into account, this statement can be seen to imply the following four claims:

(1) When people are in cyberspace, they are in an al ternative reality.

(2) People visit this alternative real ity to escape from their everyday reality.

(3) This alternative reality is not as good as everyday reality: it is trivial and glamorized and lacks context.

(4) It therefore does not constitute an adequate escape from everyday reality.

Claim 1 is mostly a conceptual claim, as it explains aspects of the meaning of the concept of "cyberspace". Claim 2 is an empirical claim about the reasons people have for visiting cyberspace, that can be investigated empirically by studying whether people actually have these reasons. Claim 3 is a normative claim (a claim which makes a value judgment), as it evaluates the quality of cyberspace relative to some normative standard. Claim 4, finally, is a logical inference from the preceding claims.

Further analysis of claim 3 must be undertaken to tease out the precise values presupposed in it. Such an analysis yields that Borgmann values authenticity and contextuality and believes that these values are not represented in cyberspace. We may then reformulate (3) to consist of the following two statements:

(3') A uthenticity and contextuality are highly valuable aspects of any reality.

(3') A uthenticity and contextuality are not present in cyberspace.

Statement 3' is a normative statement, and statement 3" an empirical statement.

A critical analysis of the two empirical claims (claims 2 and $3^{\prime \prime}$ ) can next be undertaken. Such analysis requires qualitative or quantitative empirical research (or, minimally, anecdotal evidence). Consideration of empirical evidence is likely to show that both these claims are too sweeping. Contra claim 2, many people seem to use the Internet not to escape reality but to acquire information or to interact with people or organizations that are also part of their everyday reality (Castells, 2001). At best, it can be said that the Internet is also used to escape from everyday reality, but this is only one of the reasons why it is used. Claim 3" seems to be too sweeping as well. If authentic means being sincere and serious and inclusive of real things and events rather than simulated ones, then there are many aspects of the Internet that are authentic. For instance, social interaction in cyberspace is often authentic in that it is often sincere and involves earnest interaction between real people. Context often seems to be present on the Internet as well. Both conversations and information on the Internet are often accompanied by a context that makes them intelligible to Internet users.

SIGCAS Computers and Society

V olume 36, N 0. 3, September 2006 
J ust as one can analyze and critique empirical claims concerning the Internet's benefits and harms, one can do this for normative and conceptual claims. For example, the values of authenticity and contextuality may be subjected to a critical analysis, in which it is investigated what the reasons are why these values should be held dear. As for conceptual criticism, it may be criticized whether the term "cyberspace" has a clear meaning and whether digital realities are properly analyzed as having spatial features. In this way, then, Borgmann's statement may be critically analyzed regarding the validity of its empirical, normative and conceptual claims. Although the preceding analysis is only meant to be suggestive, I hope to have shown that it is possible in general to systematically analyze and assess claims about the benefits and harms of the Internet. The uncertain factor in such assessments lies in the analysis of values, since there is no objective way in which to validate or refute values that underlie normative statements.

\section{$5 \quad$ RELATING BELIEFSABOUT THE INTERNET TO VALUE SYSTEMSAND WORLDVIEWS}

The values presupposed in people's claims about the Internet's benefits and harms may be idiosyncratic, but frequently they are part of more widely shared value systems or ideologies. A better understanding of current opinions about the Internet would for that reason be served by an understanding of how these beliefs are shaped by such value systems or ideologies. M ajor shared value systems or ideologies have formed over time, possibly over centuries, are shared by large groups of people, and have often attained some degree of institutionalization. I will call such ideologies, after Rawls (1993), comprehensive doctrines. A nother name may be worldview or ideology. Comprehensive doctrines are systems of value, be they religious, moral or ideological, that contain values concerning what is good and bad, and are often accompanied by norms for conduct and a system of (metaphysical) beliefs.

Examples of comprehensive doctrines are world religions like Christianity and Islam and their different strands, and secular humanism. Religious systems often include a transcendent conception of good and bad, according to which standards for goodness are given by a divine being. For instance, in varieties of Christianity, the highest good is the glory of God, or obedience to God's law. Secular humanists do not recognize a God, and hold that the only good is the human good, and that the highest good is individual well-being.

The rise of a consumer society has led scholars to characterize contemporary culture as a consumer culture, which carries its own set of values about what is important in life (Slater, 1997; F eatherstone, 1991). Consumerism can be defined as an ideology that holds that physical well-being and the collection and consumption of material goods is the greatest good and highest value in life. In a secularized consumer society, it can be argued, advertisers have replaced the minister in advocating a particular conception of good and bad, or they are competing with him and winning. Consumerism can therefore be considered a new comprehensive doctrine being promoted by the modern market.

Consumerism has been criticized because of its hedonism, individualism and self-interestedness, and its definition of the good life in terms of material goods, which critics have claimed should be considered instrumental goods rather than ends. Based in part on extensive empirical research, it has been argued that in the contemporary West, a new, postmaterialist doctrine is emerging in which people place greater values on ideas than on physical pleasure and material goods (Inglehart, 1990, 1997). Postmaterialists emphasize nonmaterial and nonhedonistic values like personal growth, quality leisure time, contemplation, meaningful relationships, care for the environment, social equality, and spirituality. The N ew Age movement can be seen as a manifestation of this, as well as the more recent voluntary simplicity movement, which embraces a lifestyle of lower consumption, less paid work, greater sustainability, less reliance on media technologies, and more self-reliance, which is argued to enhance the quality of life (Etzioni, 1998; Shaw and Newton, 2002).

Political ideologies, like liberalism and socialism, are usually not comprehensive doctrines, because their aim is to specify the role of the state in realizing and distributing goods, and they often do so without embody a full-blown value system that labels things and behaviors generally as good or bad. However, political ideologies often presuppose at least a partial conception of what is good or bad. For instance, communitarianism, a political ideology that holds that the state should preserve communities and should often prioritize the interests of communities over those of individuals, presupposes a limited concept of the good according to which individual well-being is dependent on the well-being of communities. Communitarians have criticized the atomistic conception of the individual in libertarianism and liberalism, which seem to hold that well-being is an individual pursuit that can be defined without reference to one's membership in a community.

SIGCA S Computers and Society

V olume 36, No. 3, September 2006 
Liberalism famously employs a "thin theory of the good" according to which the principal task of government is to create the political and economic conditions under which individuals are freely able to pursue their own conception of the good (Rawls, 1971). In other words, individuals should decide for themselves what is good or bad, as long as they leave each other free. Conservatism, finally, can be understood as an ideology that strives to preserve existing social order and the institutions that sustain it. When these institutions embody a particular conception of the good, which is often the case, conservatism may take on the form of a comprehensive doctrine that seeks to uphold a particular conception of the good. However, different conservatisms may correspond to quite different concepts of the good. In Iran or China, traditional institutions embody ideals of the good that are quite different than those in the U nited States, so conservatism in these countries also means something different.

A $n$ analysis of statements and positions regarding the Internet's benefits and harms can often be improved by recognizing the comprehensive doctrine(s) underlying these statements and positions. It is for this reason that I emphasized in section 1 that the Council of Torah Sages is operating according to the value system contained in Hareidi, a variety of orthodox Judaism, and that Esther Dyson can be (loosely) identified as a libertarian author. Such characterizations may lead to a better understanding of the reasons why individuals hold particular positions regarding the Internet. Nevertheless, it should be recognized that characterizations of comprehensive doctrines are always ideal typical descriptions that need not apply to everyone, and that particular individual may have all sorts of idiosyncratic values and beliefs that deviate from those that are typical within a particular comprehensive doctrine.

\section{6 \\ CONCLUSION: SOCIAL AND CULTURAL ASSESSMENT OF THE INTERNET}

The analysis and evaluation of claims about the Internet's benefits and harms is one step towards a general assessment of the Internet's benefits and harms. Such a general assessment would evaluate and weigh perceived harms and benefits of the Internet relative to a particular value system. Such an assessment would necessarily be subjective because of its choice of values relative to which the assessment is made. However, this certainly does not imply that such assessments are useless. If the values in question are representative of a particular social group, then the assessment can be used by the group to further its interests. The values can, however, also be the result of democratic compromise, as when different constituencies in a society or organization negotiate the values that should shape policy. In this case, the assessments can be said to be representative of the values of a whole society or of the members of an organization.

Social and cultural assessments of the Internet are useful because they can guide policies for the design, regulation and use of the Internet. Only if there is an understanding of the social and cultural implications of the Internet and the way in which these relate to one's values can one undertake effective action to cause these implications to be in agreement with one's values. Such an understanding is only a first step, however, because in addition one would need to know how to bring about change.

I have argued elsewhere that there are three basic ways in which the impacts of a technology can be changed (Brey, 1999) (M y ideas here are heavily indebted to the work of B ryan Pfaffenberger (1992)). First, it is possible to redesign or reconfigure the technology (technological delegation). M anufacturers can redesign technologies to have different design features that change the likelihood of occurrence of certain social and cultural consequences. For example, manufacturers may design Internet software to be strongly protective of privacy. Owners and users can affect what technology does by choosing particular technologies, configurations and settings over others. Such redesign and reconfiguration can be explicitly guided by values, using methods of value-sensitive design ( $\mathrm{N}$ issenbaum, 1998; Friedman, 2004).

Second, it is possible to change the way in which a technology is used or the social setting or context in which it is used (structuration). For example, one may instate particular policies or laws that regulate the use of the Internet, or one may change the structure of an organization in which the Internet is used. Third, finally, it is possible to change the way in which a technology is interpreted, understood and talked about (signification). By assigning different meanings to a technology, both denotative (regarding its form and function) and connotative (regarding its emotional and figurative meaning), it is possible to affect the way in which the technology is understood and therefore used and treated. This, in turn, may bring about different social and cultural consequences. 
I have argued in this essay that it is important to gain a better understanding of positive and negative appraisals of the Internet and to analyze and evaluate the presuppositions of such appraisals. I then provided an overview of some major claimed benefits and harms of the internet. N ext, I presented an outline of a method for analyzing statements or beliefs concerning social and cultural harms correlated with Internet use, and for relating such statements to value systems or what I have called comprehensive doctrines. Finally, I argued that assessments of (beliefs about) the Internet's benefits and harms are important to the development of policies or strategies for altering the Internet's social and cultural impacts. I outlined three basic ways, technological delegation, structuration and signification, in which these impacts can be altered. I hope to have shown, in this short space, that a better understanding of (different perspectives on) the Internet's social and cultural benefits and harms is both desirable and attainable, and that such a perspective can be used to develop better policies and strategies for steering the social and cultural consequences of the Internet.

\section{REFERENCES}

Baudrillard, J. (1995) Simulacra and Simulation (Trans. S. Glaser). University of M ichigan Press.

Borgmann, A . (1999) Holding On to Reality: The Nature of Information at the Turn of the M illennium. University of Chicago Press.

B orgmann, A . (2004) Is the Internet the Solution to the Problem of Community? In Community in the Digital Age: Philosophy and Practice. A. Feenberg \& M. Bakardjieva (eds.). Rowman \& Littlefield, Lanham, M D. 53-67.

B rey, P. (1999) W orker A utonomy and the D rama of Digital N etworks in Organizations. J ournal of Business E thics. 22: $15-25$.

Castells, M . (2001) The Internet Galaxy. Reflections on Internet, Business, and Society. Oxford U niversity Press.

Dijk, J. van (1999) The Network Society. Sage.

Dreyfus, H. (1999) A nonymity versus Commitment: the Dangers of Education on the Internet. Ethics and Information Technology. 1: 15-21.

Dreyfus, H. (2001) On the Internet. Routledge.

Dyson, E. (1997) Release 2.0. A D esign for Living in the Digital Age. B roadway B ooks, New Y ork.

Edmunds, A and M orris, A . (2000) The Problem of Information O verload in Business Organisations: A R eview of the Literature. International J ournal of Information Management. 20: 17-28.

Ess, C. and Sudweeks, F. (2001) Culture, Technology, Communication: Towards an Intercultural Global Village. State U niversity of N ew Y ork Press, N ew Y ork.

Etzioni, A . (1998) V oluntary Simplicity: Characterization, Select Psychological Implications, and Societal Consequences. J ournal of Economic P sychology. 19: 619-43.

Featherstone, M. (1991) Consumer Culture and Postmodernism. Sage, London.

Friedman, B. (2004) V alue-Sensitive D esign. Encyclopedia of H uman-C omputer Interaction. Berkshire Publishing Group, Great B arrington, M A. 769-774.

Inglehart, R. (1990) Culture Shift in Advanced Industrial Society. Princeton U niversity Press, Princeton, NJ .

Inglehart, R. (1997) Modernization and Postmodernization: Cultural, Economic, and Political Change in 43 Societies. Princeton U niversity Press, Princeton, NJ .

M cCormick, M. (2001) Is It W rong to Play V iolent Video Games? Ethics and Information Technology. 3: 277-287.

Nissenbaum, H. (1998) V alues in the Design of Computer Systems. Computers in Society. 38-39.

Pfaffenberger, B. (1992) Technological Dramas. Science, Technology and H uman Values. 17: 282-312.

Rawls, J. (1971) A Theory of J ustice. Harvard U niversity Press, Cambridge, M A .

Rawls, J. (1993) Political Liberalism. Columbia U niversity Press.

Rheingold, H. (2000) The Virtual Community: Homesteading on the Electronic F rontier, revised edition. M IT Press, Cambridge.

Shaw, D. and N ewholm, T. (2002) V oluntary Simplicity and the Ethics of Consumption. Psychology and M arketing. 19 (2): 167-185.

Slater, D. (1997) Consumer Culture and Modernity. Polity Press.

Turkle, S. (1995) Life on the Screen: Identity in the Age of the Internet. Simon \& Schuster, N ew Y ork.

W inner, L. (1997) Cyberlibertarian M yths and the Prospects for Community. Computers and Society, 27 (3): 14-19.

Y oung, K. (2004) Internet A ddiction: A N ew Clinical Phenomenon and its Consequences. The American Behavioral Scientist, 48(1): 402-415. 